so that the relation (3.1) is an obvious consequence of (3.2), (3.3), (3.4), and (3.8).

\title{
REFERENCES
}

1. F. Riesz, Les systèmes d'équations lineaires d une infinité d'inconnues, Paris, 1913.

2. I. J. Schoenberg, Some analytical aspects of the problem of smoothing, Courant Anniversary Volume, New York, 1948, pp. 351-370.

3. G. Szegö, Beiträge zur Theorie der Toeplitzschen Formen, Math. Zeit. vol. 6 (1920) pp. 167-202.

UNIVERSITY OF Colorado AND

STANFORD UNIVERSITY

\section{A NEUMANN SERIES FOR THE PRODUCT OF TWO WHITTAKER FUNCTIONS'}

\section{PETER HENRICI}

1. Introduction. Several series representations in terms of Bessel functions have been given recently for a single Whittaker function of the first kind (usually denoted by $M_{k, \mu}(z)$ ) by various authors. ${ }^{2}$ None of these series is very easy to survey, because their coefficients are never given explicitly but only either by means of a generating function (as in [4]) or by recurrence formulae (as in [1] and [6]). In the present paper a generalized Neumann series for the product of two Whittaker functions of the first kind with common indices but different arguments is given, in which the coefficients are formed by certain terminating generalized hypergeometric series and by Gegenbauer polynomials. The expansion obtained includes also the case of a single Whittaker function, which is of special interest in connection with the theory of Coulomb waves. ${ }^{3}$ Our method of proof uses only the simplest properties of the function $M_{x, \mu}(z)$ and is especially much more elementary than the methods of [4]. All symbols used are those of Magnus-Oberhettinger [7], with exception of that for the generalized hypergeometric series, for which we use Bailey's [2] notation.

2. Theorem. For arbitrary complex values or $r, \theta, k$, and $4 \mu$ $\neq-1,-2, \cdots$ the following expansion holds:

Received by the editors May 26, 1952.

1 This paper was prepared in part under a National Bureau of Standards Contract with The American University.

2 Abramowitz [1], Buchholz [4], Karlin [6], H. Schmidt [4], Tricomi [4].

${ }^{3} \mathrm{Cf}$. [1] and the literature quoted there. The function $\phi_{L}(\eta, \rho)$ considered in [1] is identical with $M_{i m L+1 / 2}(2 i \rho) \cdot(2 i \rho)^{-(L+1)}$. 
(1)

$$
\begin{gathered}
M_{\kappa, \mu}(i r(1+\cos \theta)) M_{\kappa, \mu}(-i r(1-\cos \theta))(r \sin \theta)^{-(2 \mu+1)} \\
=2^{2 \mu+1 / 2} \Gamma\left(2 \mu+\frac{1}{2}\right) \sum_{n=0}^{\infty} \frac{(-i)^{n}(2 \mu+1 / 2+n)(\mu+\kappa+1 / 2)_{n}}{(2 \mu+1)_{n}} \\
\times{ }_{2} F_{2}\left[\begin{array}{l}
\mu+1 / 2+\kappa,-2 \mu-n,-n ; \\
-\kappa+1 / 2-\mu-n, 2 \mu+1 ;
\end{array}\right] r^{-(2 \mu+1 / 2)} J_{2 \mu+1 / 2+n}(r) \\
\cdot C_{n}^{(2 \mu+1 / 2)}(\cos \theta) .
\end{gathered}
$$

3. Proof.

3.1. Systems of coordinates. The following considerations are made in the two-dimensional complex Euclidean $(x, y)$-space $K^{2}$. We shall also use in this space polar coordinates $(r, \theta)$ and parabolic coordinates $(\xi, \eta)$, respectively connected with $(x, y)$ by the relations

$$
\begin{aligned}
& x=r \cos \theta=\left(\xi^{2}-\eta^{2}\right) / 2, \\
& y=r \sin \theta=\xi \eta .
\end{aligned}
$$

The ambiguities arising by (2) in the complex part of $K^{2}$ will not be of significance in the following. Evidently, from (2),

$$
\xi^{2}=r(1+\cos \theta), \quad \eta^{2}=r(1-\cos \theta) .
$$

A fourth system of coordinates will be introduced in 3.4.

3.2. Differential equation satisfied by (1). It is well known [3] that, provided $2 \mu \neq-1,-2, \cdots$, the function

$$
v(x, y)=(\xi \eta)^{-1} M_{\alpha, \mu}\left(i \xi^{2}\right) M_{k, \mu}\left(-i \eta^{2}\right)
$$

(not identical with (1)) satisfies as function of $x$ and $y$ the equation

$$
\frac{\partial^{2} v}{\partial x^{2}}+\frac{\partial^{2} v}{\partial y^{2}}+\frac{1}{y} \frac{\partial v}{\partial y}+\left(1-\frac{4 \mu^{2}}{y^{2}}\right) v=0 .
$$

From here it is easily verified that $u(x, y)=y^{-2 \mu} v(x, y)$, i.e. the lefthand side of (1) satisfies

$$
\frac{\partial^{2} u}{\partial x^{2}}+\frac{\partial^{2} u}{\partial y^{2}}+\frac{2 \nu}{y} \frac{\partial u}{\partial y}+u=0,
$$

where $\nu=2 \mu+1 / 2.4$

3.3. Neumann series expansions for solutions of (4). It is generally assumed $^{5}$ and can easily be proved (although an explicit proof does

\footnotetext{
- We need not consider here the well known physical interpretation of the equations (3) and (4).

Cf. Watson [8, p. 367], and the papers of Gegenbauer quoted there.
} 
not seem to have been ever given), that if $\nu \neq 0,-1,-2, \cdots$, any solution $w(x, y)$ of $(4)$, which is regular near $x=y=0$, can be expanded there in a unique way into a (generalized) Neumann series of normal solutions of (4) as follows:

$$
w(x, y)=\sum_{n=0}^{\infty} a_{n} r^{-r} J_{v+n}(r) C_{n}^{(v)}(\cos \theta) .
$$

In case $w$ is an entire function of $x$ and $y,(5)$ converges for arbitrary $x$ and $y$. Assuming this result here and taking in account that $u$ is an entire function of $x$ and $y$, the existence of an expansion of $u$ of the form (5) (and thus (1)) is established, and there remains only the determination of the coefficients $a_{n}$ in (5), when $w=u$.

3.4. Characteristic coordinates. To this purpose we introduce in (5) the new variables ${ }^{6}$

$$
z=x+i y, \quad z^{*}=x-i y .
$$

We have then $r^{2}=z z^{*}, \cos ^{2} \theta=\left(z+z^{*}\right)^{2} / 4 z z^{*}$ and therefore

$$
r^{-(v+n)} J_{v+n}(r)=2^{-(v+n)} \sum_{m=0}^{\infty} \frac{\left(-z z^{*} / 4\right)^{m}}{m ! \Gamma(\nu+n+m+1)}
$$

and

$$
r^{n} C_{n}^{(\nu)}(\cos \theta)=\frac{\Gamma(\nu+n)}{n ! \Gamma(\nu)}\left(z+z^{*}\right)^{n}
$$

$$
\cdot{ }_{2} F_{1}\left(-\frac{n}{2},-\frac{n}{2}+\frac{1}{2}, 1-\nu-n ; \frac{4 z z^{*}}{\left(z+z^{*}\right)^{2}}\right) \text {, }
$$

further

$$
\xi^{2}=\left(z^{1 / 2}+z^{* 1 / 2}\right)^{2} / 2, \quad \eta^{2}=-\left(z^{1 / 2}-z^{* 1 / 2}\right)^{2} / 2 .
$$

3.5. Comparison of coefficients. As (5) holds for arbitrary values of $z$ and $z^{*}$, it holds for $z^{*}=0$. It follows from (7) and (8) that in this case the right-hand side of $(5)$ reduces to

$$
\sum_{n=0}^{\infty} a_{n} \frac{\Gamma(\nu+n)}{2^{\nu+n} \Gamma(\nu+n+1) \Gamma(\nu) n !} z^{n}
$$

For $u(x, y)$ we obtain by the definition of $M_{x, \mu}(z)$, by

$$
M_{\alpha, \mu}(z)=M_{\rightarrow, \mu}\left(e^{i \pi} z\right) e^{-i \pi(\mu+1 / 2)},
$$

- The symbols $x$ and $y$ are treated here as independent complex variables. Therefore, in general, $\bar{z} \neq z^{*}$. 
by (9), and due to $\xi^{2}+\eta^{2}=2 r=0$,

$$
\begin{aligned}
{ }_{1} F_{1} & \left(-\kappa+\mu+\frac{1}{2} ; 2 \mu+1 ; \frac{i z}{2}\right) \\
& \cdot{ }_{1} F_{1}\left(\kappa+\mu+\frac{1}{2} ; 2 \mu+1 ;-\frac{i z}{2}\right) \\
= & \sum_{n=0}^{\infty}\left(\frac{-i z}{2}\right)^{n} \sum_{m=0}^{n} \frac{(-1)^{m}(\mu+\kappa+1 / 2)_{n-m}(\mu-\kappa+1 / 2)_{m}}{(2 \mu+1)_{n-m}(2 \mu+1)_{m}(n-m) ! m !} \\
= & \sum_{n=0}^{\infty}\left(\frac{-i z}{2}\right)^{n} \frac{(\mu+\kappa+1 / 2)_{n}}{n !(2 \mu+1)_{n}} \\
& \cdot{ }_{3} F_{2}[-2 \mu-n, \mu-\kappa+1 / 2,-n ; 1] .
\end{aligned}
$$

Comparing the coefficients of $z^{n}$ in (10) and (11) and remembering the definition of $\nu$, we get, denoting the generalized hypergeometric series in (11) simply by ${ }_{3} F_{2}$,

$$
a_{n}=2^{2 \mu+1 / 2} \Gamma(2 \mu+1 / 2) \frac{(2 \mu+1 / 2+n)(\mu+\kappa+1 / 2)_{n}}{(2 \mu+1)_{n}}(-i)^{n_{3}} F_{2} .
$$

Inserting this in (5), we obtain (1).

4. Special cases. Among the various expansions, which may be derived from (1) as special cases, the following are of particular interest.

4.1. Expansion of a single $M_{\kappa, \mu}$. Because

$$
C_{n}^{(v)}(1)=\frac{\Gamma(2 \nu+n)}{\Gamma(2 \nu) n !}
$$

$\theta=0$ in (1) yields immediately

$$
\begin{aligned}
& M_{\kappa, \mu}(2 i r) \cdot(2 i r)^{-(\mu+1 / 2)}=2^{2 \mu+1 / 2} \Gamma(2 \mu+1 / 2) \\
& \times \sum_{n=0}^{\infty} \frac{(-i)^{n}(2 \mu+1 / 2+n)(\mu+\kappa+1 / 2)_{n}(4 \mu+1)_{n}}{(2 \mu+1)_{n} n !}{ }_{3} F_{2} r^{-(2 \mu+1 / 2)} J_{2 \mu+1 / 2+n}(r) .
\end{aligned}
$$

4.2. Product of Bessel functions. If we put $\kappa=0$ in (1), the Whittaker functions degenerate $[9$, p. 338] into certain Bessel functions. On the other hand,

$$
{ }_{3} F_{2}={ }_{3} F_{2}\left[\begin{array}{ll}
\mu+1 / 2,-2 \mu-n,-n ; & 1 \\
1 / 2-\mu-n, 2 \mu+1 ; &
\end{array}\right]
$$


becomes then well-poised $[2$, p. 13] and can therefore be expressed in terms of $\Gamma$-functions. Thus we obtain, after some manipulation, the expansion

$$
\begin{aligned}
J_{\mu} & \left((1+\cos \theta) \frac{r}{2}\right) J_{\mu}\left(\frac{r}{2}(1-\cos \theta)\right)(r \sin \theta)^{-2 \mu} \\
= & \left(\frac{2}{\pi}\right)^{1 / 2} \Gamma(2 \mu+1 / 2) \\
& \cdot \sum_{m=0}^{\infty}(-1)^{m}(2 \mu+1 / 2+2 m)\left(\frac{1}{2}\right)_{m} \frac{\Gamma(1 / 2+\mu+m)}{\Gamma(1+2 \mu+m) \Gamma(1+\mu+m)} \\
& \cdot r^{-(2 \mu+1 / 2)} J_{2 \mu+1 / 2+2 m}(r) C_{2 m}^{(2 \mu+1 / 2)}(\cos \theta),
\end{aligned}
$$

also valid only provided $4 \mu \neq-1,-2, \cdots$. This result can also be established directly the method of 3 .

4.3. Gegenbauer's expansion. In the case $\kappa=\mu+1 / 2$ we have [9, p. 338], $M_{\mu+1 / 2, \mu}(z)=z^{\mu+1 / 2} e^{-z / 2}$. At the same time the ${ }_{3} F_{2}$ in (1) reduces to 1 . We obtain thus immediately the following well known generalization (due to Gegenbauer) of the Jacobi-Anger formula:

$$
\begin{aligned}
e^{-i r \cos \theta}=2^{\nu} \Gamma(\nu) \sum_{n=0}^{\infty}(-i)^{n}(\nu+n) r^{-\nu} J_{\nu+n}(r) C_{n}^{(\nu)}(\cos \theta), & \\
\nu & \neq-1,-2, \cdots .
\end{aligned}
$$

5. Remarks. The proof of (1), given (with exception of the remark at the beginning of 3.3) in full rigor can be rendered intuitive in the following way. Introducing the variables (6) into (4), we obtain the equation

$$
\frac{\partial^{2} u}{\partial z \partial z^{*}}-\frac{2 \mu+1 / 2}{z-z^{*}}\left(\frac{\partial u}{\partial z}-\frac{\partial u}{\partial z^{*}}\right)+\frac{1}{4} u=0,
$$

i.e. a hyperbolic equation, whose two families of characteristics are given by $z=$ const., $z^{*}=$ const. respectively. Putting $z^{*}=0$ in (5) means therefore that we are considering both sides of (5) on a characteristic. Now it is a well known fact that (vaguely speaking) the solution of a hyperbolic equation in two variables is determined by its values on one characteristic of each family. Moreover, since $u$ is symmetric in $z$ and $z^{*}$, it suffices here to consider the values on only one characteristic. Any relation between solutions of (4) or (12) can therefore be proved by considering the values on a characteristic 
only. The outlined method yields in fact also very rapid proofs, e.g. for the addition theorems of the Bessel functions. ${ }^{7}$

\section{BIBLIOGRAPHY}

1. M. Abramowitz, Coulomb wave functions expressed in terms of Bessel-Clifford and Bessel functions, J. Math. Phys. vol. 29 (1950) pp. 303-308.

2. W. N. Bailey, Generalized hypergeometric series, Cambridge, 1935.

3. H. Buchholz, Die konfluente hypergeometrische Funktion etc., ZAMM vol. 23 (1943) pp. 47-58.

4. - Komplexe Integrale fuer die parabolischen Funktionen etc., Math. Zeit. vol. 53 (1950) pp. 387-402.

5. P. Henrici, Bergman's Integraloperator erster Art und Riemannsche Funktion, Zeitschrift fuer angewandte Mathematik und Physik vol. 3 (1952) pp. 228-232.

6. M. Karlin, Note on the expansion of confluent hypergeometric functions in terms of Bessel functions of integral order, J. Math. Phys. vol. 28 (1949) pp. 43-44.

7. W. Magnus and F. Oberhettinger, Formeln und Saetze fuer die speziellen Funktionen der mathematischen Physik, 2d ed., Berlin, 1948.

8. G. N. Watson, $A$ treatise on the theory of Bessel functions, 2d ed., Cambridge, 1944.

9. E. T. Whittaker and G. N. Watson, $A$ course of modern analysis, 4th ed., Cambridge, 1927.

\section{American University}

7 A short discussion of the application of hyperbolic equation methods to elliptic equations with regular coefficients is given in [5]. 